\title{
Set-membership LPV model identification of vehicle lateral dynamics ${ }^{\star}$
}

\author{
Vito Cerone ${ }^{\S}$, Dario Piga, Diego Regruto \\ Dipartimento di Automatica e Informatica, Politecnico di Torino, Corso Duca degli Abruzzi 24, 10129 Torino, Italy
}

\begin{abstract}
Set-membership identification of a Linear Parameter Varying (LPV) model describing the vehicle lateral dynamics is addressed in the paper. The model structure, chosen as much as possible on the ground of physical insights into the vehicle lateral behavior, consists of two single-input single-output LPV models relating the steering angle to the yaw rate and to the sideslip angle. A set of experimental data obtained by performing a large number of manoeuvres is used to identify the vehicle lateral dynamics model. Prior information on the error bounds on the output and the time-varying parameters measurements are taken into account. Comparison with other vehicle lateral dynamics models is discussed.
\end{abstract}

Key words: Bounded error, Linear Parameter Varying, Set-Membership, Uncertain scheduling variables, Vehicle lateral dynamics.

\section{Introduction}

A remarkable number of vehicle dynamics control systems have been proposed in the last decades to effectively improve driving safety, vehicle handling and passenger comfort. Lane keeping systems (Suryanarayanan et al., 2004; Cerone et al., 2009a), active (Rajamani and Hedrick, 1994) and semiactive (Poussot-Vassal et al., 2008) suspension systems, adaptive cruise control (Rajamani et al., 2000) are just a few examples. Due to the interest in these control systems, modeling of road vehicle behavior has received a renewed attention in recent years. Indeed, the plant mathematical model should be carefully selected in order to design effective control systems, paying also attention to the tradeoff between model complexity and accuracy.

The most popular among the vehicle lateral dynamics models proposed in the literature is a linear second order model, referred to as single track (or bicycle) model (see, e.g., Rajamani (2006) for details). The linear single track model is used in many papers to design linear controllers of the lateral dynamics both for lane keeping (Cerone et al., 2009a; Suryanarayanan et al., 2004) and

\footnotetext{
‡ This paper was not presented at any IFAC Conference. $\S$ Corresponding author V. Cerone. Tel. +39-(0)11-5647064 Fax +39-(0)11-5647064

Email addresses: vito.cerone@polito.it (Vito Cerone), dario.piga@polito.it (Dario Piga), diego.regruto@polito.it (Diego Regruto).
}

yaw stability applications (Cerone et al., 2009b; Güvenç et al., 2004). However, such a simple model becomes inadequate when either the longitudinal velocity is allowed to vary rapidly or aggressive steering manoeuvres like steering angle steps or double lane change manoeuvres, are performed. In such cases, more complex models are needed. A good deal of remarkable works has been recently focused on the problem of model based on-line estimation of some key variables of the vehicle lateral dynamics. These estimators, sometimes called virtual sensors, can be profitably used to replace physical sensors which cannot be mounted on production cars due to their prohibitive costs. In (Piyabongkarn et al., 2009) the authors discuss an effective algorithm for sideslip angle estimation based on a proper combination of the estimates provided by a linear single track model and a kinematics model. The problem of sideslip angle estimation is also considered in (Stephant et al., 2004) where a linear estimator based on the single track model is compared with three different nonlinear filters designed on the basis of some proper nonlinear extensions of the bicycle model. The obtained results show that the nonlinear observers significantly outperform the linear one. The papers mentioned above motivate the development of nonlinear models of the lateral dynamics.

In recent years, the linear parameter varying (LPV) modeling approach received a major attention from the identification and control research community, mainly due to the strong connection between LPV models and gain scheduling control design methods (see the survey 
paper Rugh and Shamma (2000) for a review of the literature on the subject). LPV models belong to the more general class of linear time-varying models and, roughly speaking, they can be defined as linear systems where, either the matrices of the state equations or the coefficients of the input-output relation, depend on one or more time varying parameters, whose real-time samples are assumed to be available. A grey-box approach to LPV modeling of vehicle dynamics is considered in (Gaspar et al., 2008). As to black-box identification of LPV models, a relevant number of approaches has appeared in the literature in the last decade. An up-to-date overview of the available LPV modeling and identification approaches can be found in the book (Toth, 2010). Most of the works on LPV identification are based on the assumption that the measurement error is statistically described. A worthwhile alternative to the stochastic description of measurement errors is the boundederror or set-membership (SM) characterization, where uncertainties are assumed to belong to a given set. Further details on this approach can be found in the book Milanese et al. (1996). Only few contributions can be found in the literature addressing the identification of LPV models when the measurement errors are supposed to be bounded. In (Sznaier and Mazzarro, 2003; Bianchi and Sanchez-Pena, 2010) identification and model invalidation of LPV systems in presence of bounded noise and a possible nonparametric part is considered. Belforte and Gay (Belforte and Gay, 2004) consider the identification of discrete-time LPV models with finite impulse response structure and output measurements affected by bounded noise.

In this paper SM identification of a black-box inputoutput LPV model of the vehicle lateral dynamics is addressed. The proposed model consists of two single input single output (SISO) LPV representations, relating the steering angle to the yaw rate and the sideslip angle respectively. The paper is organized as follows. The selection of the two SISO models structure is discussed in Section 2 relying on physical insights about the vehicle lateral behavior. Then, the approach for the identification of SISO discrete-time LPV models when both the output and the time-varying parameters measurements are affected by bounded noise, previously presented by the authors in (Cerone and Regruto, 2008), is briefly reviewed and extended in Section 3. In Section 4 experimental data obtained by performing a large number of different manoeuvres are exploited to identify a blackbox LPV vehicle lateral dynamics model through the algorithm described in Section 3. The performances of the identified LPV model are compared with the ones obtained by other vehicle dynamics models.

\section{LPV model of the lateral dynamics: structure selection}

The aim of our contribution is to obtain a model which adequately describes the input-output relations between the steering angle and the two most relevant variables of the lateral dynamics, i.e., the yaw rate and the sideslip angle. Therefore, the proposed system description consists of two SISO LPV models, relating the steering angle to the yaw rate and to the sideslip angle respectively. The choice of an appropriate model structure should be based as much as possible on insight and knowledge about the system to be identified. To this aim, in this section the fundamental aspects of vehicle lateral dynamics are briefly reviewed. Then, physical insights are used to select the scheduling variables and the dynamic order of the two SISO LPV models to be identified.

\subsection{Brief review of vehicle lateral dynamics}

A single track (or bicycle) model with two degrees of freedom is considered here to describe the vehicle behavior, according to (Rajamani, 2006; Piyabongkarn et al., 2009). Among the possible equivalent choices for the model state variables, we consider the body sideslip angle $\beta$ and the yaw rate $r$ since they are of particular interest when describing the vehicle lateral dynamics, which, under the assumption of negligible road bank angle, can be described by the following two equations (see, e.g., Rajamani, 2006)

$$
m V_{x}(\dot{\beta}+r)=F_{y_{f}}+F_{y_{r}}, I_{z} \dot{r}=l_{f} F_{y_{f}}-l_{r} F_{y_{r}}
$$

where $m$ is the vehicle mass, $I_{z}$ is the yaw moment of inertia, $V_{x}$ is the vehicle longitudinal velocity, $F_{y_{r}}$ is the rear lateral tire force, $F_{y_{f}}$ is the front tire lateral force, $l_{r}$ and $l_{f}$ are the distances between the projection of the vehicle center of mass to the ground and the tire-road contact point in static conditions. Under the assumption of small tires slip angles, front and rear lateral forces can be reasonably described by

$$
F_{y_{f}}=c_{f} \alpha_{f}, \quad F_{y_{r}}=c_{r} \alpha_{r}
$$

where $\alpha_{f}$ and $\alpha_{r}$ are the front and the rear tire slip angles respectively, defined as the angles between the tire longitudinal axes and the directions of the tire velocity vectors. These angles can be related to the state variables $\beta$ and $r$ through

$$
\alpha_{f}=\delta-\beta-r l_{f} / V_{x}, \quad \alpha_{r}=-\beta+r l_{r} / V_{x},
$$

where $\delta$ is the steering angle. Substitution of equations (2) - (3) into equations (1) leads to the first order nonlinear differential equations

$$
\dot{r}=-\beta\left(\frac{l_{f} c_{f}}{I_{z}}-\frac{l_{r} c_{r}}{I_{z}}\right)-r\left(\frac{l_{f}^{2} c_{f}}{I_{z} V_{x}}+\frac{l_{r}^{2} c_{r}}{I_{z} V_{x}}\right)
$$




$$
\begin{aligned}
\dot{\beta}= & -\beta\left(\frac{c_{f}}{m V_{x}}+\frac{c_{r}}{m V_{x}}\right)-r\left(1-\frac{l_{f} c_{f}}{m V_{x}^{2}}+\frac{l_{r} c_{r}}{m V_{x}^{2}}\right)+ \\
& +\frac{c_{f}}{m V_{x}} \delta .
\end{aligned}
$$

Under the assumption of constant longitudinal velocity $V_{x}$, equations (4) and (5) provide a second order linear time-invariant model. In spite of its simplicity, such a linear model provides a good tradeoff between complexity of the model structure and accuracy in describing the vehicle behavior. However, the accuracy of the linear bicycle model significantly decreases in the presence of time-varying longitudinal velocity $V_{x}(t)$. Furthermore, when aggressive steering manoeuvres exciting the tires nonlinear behavior are performed, lateral forces can no more be described by equation (2). In order to overcome such drawbacks, in this paper an LPV model for the vehicle lateral dynamics is introduced and identified by exploiting experimental data.

\subsection{Lateral dynamics model structure}

In order to take into account the key features of the vehicle lateral dynamics discussed in Section 2.1, here we address the problem of selecting the structure of the SISO black-box LPV models to be identified on the basis of the following considerations:

- second order difference equations are considered to retain information on the dynamics order of the single track physical model;

- to take into account the effects of possible timevarying longitudinal velocity, nonlinear dependence on the reciprocal of the longitudinal velocity is explicitly considered in the coefficients of the difference equations;

- since the nonlinear tire behavior is excited by aggressive steering manoeuvres, which in turn lead to large lateral acceleration values, nonlinear dependence on lateral acceleration is explicitly considered in the coefficients of the difference equations.

Based on these considerations, we introduce the following SISO LPV models to describe the vehicle dynamics:

$$
\begin{gathered}
r_{t}+\sum_{i=1}^{2} a_{i}\left(V_{x_{t}}^{-1}, a_{y_{t}}\right) r_{t-i}=\sum_{j=0}^{2} b_{j}\left(V_{x_{t}}^{-1}, a_{y_{t}}\right) \delta_{t-j} \\
\beta_{t}+\sum_{i=1}^{2} c_{k}\left(V_{x_{t}}^{-1}, a_{y_{t}}\right) \beta_{t-k}=\sum_{\nu=0}^{2} d_{\nu}\left(V_{x_{t}}^{-1}, a_{y_{t}}\right) \delta_{t-\nu},
\end{gathered}
$$

where $r_{t}, \beta_{t}, V_{x_{t}}, a_{y_{t}}$ and $\delta_{t}$ are the samples at time $t$ of the yaw rate, body sideslip angle, longitudinal velocity, lateral acceleration and steering angle respectively. Coefficients $a_{i}, b_{j}, c_{k}, d_{\nu}$ are assumed to be polynomial memoryless functions of parameters $a_{y_{t}}$ and $V_{x_{t}}^{-1}$.

Remark 1 - It is worthwhile pointing out that our objective is to model the vehicle lateral dynamics from an input-output point of view, thus we are not interested in estimating the value of the physical parameters of the vehicle. Therefore, we have chosen to describe the system under consideration by means of two black-box models whose parameters are not related with those involved in the single track model reviewed in Section 2.1.

Remark 2 - Lateral acceleration measurements can be obtained through low cost accelerometers, while many different effective approaches can be found in the literature to compute a reliable estimate of the vehicle longitudinal velocity (see, e.g., Imsland et al., 2006) using information from on-board sensors commonly available on production cars. Availability of such low cost measurements of the scheduling variables makes it possible to use the proposed LPV models in real-time applications such as, for example, virtual sensors for on-line estimation of sideslip angle and yaw rate.

Remark 3 - The proposed model structure does not take explicitly into account the dependency on the surface adhesion coefficient. Since the lateral dynamics strongly depends on the surface, we suggest to identify a bank of LPV models each one corresponding to a different value of the adhesion coefficient. Scheduling among the models in the bank, can be performed in real-time on the basis of the adhesion coefficient value estimated with one of the algorithm available in the literature like the one presented in (Wang et al., 2004).

\section{Identification procedure}

In this section we review the algorithm proposed in (Cerone and Regruto, 2008) for the identification of SISO discrete time LPV models when both the output and the time-varying parameters are affected by bounded noise.

\subsection{Formulation of the identification problem}

Consider the SISO discrete-time LPV model described in terms of a linear difference equation

$$
w_{t}+\sum_{i=1}^{n a} \alpha_{i}\left(\lambda_{t}\right) w_{t-i}=\sum_{j=0}^{n b} \beta_{j}\left(\lambda_{t}\right) u_{t-j}
$$

where $u_{t}$ and $w_{t}$ are the input and the output signals respectively, $\lambda_{t} \in \mathbb{R}^{\mu}, \lambda_{t}=\left[\lambda_{1_{t}} \lambda_{2_{t}} \ldots \lambda_{\mu_{t}}\right]^{\mathrm{T}}$ is a vector of time-varying parameters which are assumed to be measurable and coefficients $\alpha_{i}$ and $\beta_{j}$ are assumed to be 
memoryless mappings of parameters $\lambda_{t}$ described by

$$
\alpha_{i}\left(\lambda_{t}\right)=\sum_{k=1}^{n_{i}} \alpha_{i, k} f_{i, k}\left(\lambda_{t}\right), \quad \beta_{j}\left(\lambda_{t}\right)=\sum_{h=0}^{m_{j}} \beta_{j, h} g_{j, h}\left(\lambda_{t}\right),
$$

where $f_{i, k}(\cdot)$ and $g_{j, h}(\cdot)$ are known nonlinear basis functions. Let $y_{t}$ and $z_{t}$ be noise-corrupted measurements of $w_{t}$ and $\lambda_{t}$ respectively

$$
y_{t}=w_{t}+\eta_{t}, \quad z_{t}=\lambda_{t}+\varepsilon_{t},
$$

with $\varepsilon_{t}=\left[\varepsilon_{1_{t}} \varepsilon_{2_{t}} \ldots \varepsilon_{\mu_{t}}\right]^{\mathrm{T}}$. Measurement uncertainties $\eta_{t}$ and $\varepsilon_{s_{t}}, s=1 \ldots \mu$, are known to range within given bounds $\Delta \eta_{t}$ and $\Delta \varepsilon_{s_{t}}$, more precisely

$$
\left|\eta_{t}\right| \leq \Delta \eta_{t}, \quad \varepsilon_{t} \in \mathcal{E}_{t},
$$

where $\mathcal{E}_{t}=\left\{\varepsilon_{t} \in \mathbb{R}^{\mu}:\left|\varepsilon_{s_{t}}\right| \leq \Delta \varepsilon_{s_{t}}, s=1,2, \ldots, \mu\right\}$. The unknown parameter vector $\theta \in \mathbb{R}^{p}$ to be estimated is defined as

$$
\begin{aligned}
\theta^{\mathrm{T}}= & {\left[\alpha_{1,1} \ldots \alpha_{1, n_{1}} \ldots \alpha_{n a, 1} \ldots \alpha_{n a, n_{n a}}\right.} \\
& \left.\beta_{0,1} \ldots \beta_{0, m_{1}} \ldots \beta_{n b, 1} \ldots \beta_{n b, m_{n b}}\right],
\end{aligned}
$$

where $p=\sum_{i=1}^{n a} n_{i}+\sum_{j=0}^{n b} m_{j}$. In the set-membership context, all parameter vectors belonging to the so-called feasible parameter set (FPS), i.e. parameters consistent with measurements, error bounds and the assumed model structure, are feasible solutions to the identification problem. Given $N$ samples of signals $u_{t}, y_{t}$ and $z_{t}$, the feasible parameter set for the LPV system described by equations $(8)-(10)$, is defined as

$$
\begin{aligned}
& \mathcal{D}_{\theta}=\left\{\theta \in \mathbb{R}^{p}: y_{t}-\eta_{t}+\right. \\
& +\sum_{i=1}^{n a} \alpha_{i}\left(z_{t}-\varepsilon_{t}\right)\left[y_{t-i}-\eta_{t-i}\right]=\sum_{j=0}^{n b} \beta_{j}\left(z_{t}-\varepsilon_{t}\right) u_{t-j} \\
& \left.\left|\eta_{t}\right| \leq \Delta \eta_{t} ; \varepsilon_{t} \in \mathcal{E}_{t} ; t=1, . ., N\right\}
\end{aligned}
$$

As discussed in (Cerone and Regruto, 2008), the exact feasible parameter region $\mathcal{D}_{\theta}$ is, in general, a noncovex set described by nonlinear inequalities whose shape may become fairly complex for increasing values of $N$. Thus, in (Cerone and Regruto, 2008) a polytopic outer approximation $\mathcal{D}_{\theta}^{\prime}$ of the exact feasible parameter set $\mathcal{D}_{\theta}$ is derived. By exploiting such a polytopic description, two different procedures based on convex optimization are proposed in Section 3.2 to select, among the elements of $\mathcal{D}_{\theta}^{\prime}$, a single model which minimizes a suitable functional of the fitting error.

\subsection{Pointwise estimators for the selection of a single model belonging to the feasible set}

Although $\mathcal{D}_{\theta}$ is the set of all the LPV models that are consistent with experimental data and error bounds, nei- ther the feasible parameter set nor its convex approximation $\mathcal{D}_{\theta}^{\prime}$ derived in (Cerone and Regruto, 2008) can be easily used either to simulate the vehicle behavior or to design a controller of the vehicle lateral dynamics. Thus, the problem of selecting a single model among the feasible ones arises. Two different pointwise estimators are considered here, both based on the minimization of the functional

$$
J(\theta)=\|\phi \theta-y\|_{2}
$$

where $\phi \in \mathbb{R}^{N \times p}$ is the regressor matrix, $y=$ $\left[y_{1} y_{2} \ldots y_{N}\right]^{\mathrm{T}}$ and $\|\cdot\|_{2}$ is the quadratic norm of a sequence. The functional $J$ is exactly the same functional minimized by the well known least-squares (LS) estimator whose optimality and robustness properties have been widely studied also in the set-membership framework (Kacewicz et al., 1986). Unlike the standard least squares estimator, which looks for the optimal solution exploring the entire $p$-dimensional parameter space, here we are interested in restricting the search among the parameters values which belong to the outer approximation $\mathcal{D}_{\theta}^{\prime}$ of the feasible set proposed in (Cerone and Regruto, 2008). Note that the standard least squares estimate is not guaranteed to be consistent with the experimental data and the assumed error bounds. Besides, since $\phi$ and $y$ are corrupted by bounded uncertainties $\varepsilon$ and $\eta$ respectively, at least in principle we have to look for the solution of the so-called robust least squares problem which consists in the minimization of the worst-case residual against the uncertainty affecting the data. Summarizing, the computation of the robust least squares estimate constrained to the set $\mathcal{D}_{\theta}^{\prime}$ leads to the solution of the optimization problem

$$
\theta^{*}=\arg \min _{\theta \in \mathcal{D}_{\theta}^{\prime}} \max _{\varepsilon \in E, \eta \in \chi} \tilde{J}(\theta, \varepsilon, \eta)
$$

where $\tilde{J}(\theta, \varepsilon, \eta)=\|\phi(\varepsilon, \eta) \theta-y(\eta)\|_{2}, \varepsilon=\left[\varepsilon_{1}^{\mathrm{T}} \varepsilon_{2}^{\mathrm{T}} \ldots \varepsilon_{N}^{\mathrm{T}}\right]^{\mathrm{T}}$, $E=\left\{\varepsilon: \varepsilon_{t} \in \mathcal{E}_{t}, t=1, \ldots, N\right\}, \eta=\left[\eta_{1} \eta_{2} \ldots \eta_{N}\right]^{\mathrm{T}}$ and $\chi=\left\{\eta:\left|\eta_{t}\right| \leq \Delta \eta_{t}, t=1, \ldots, N\right\}$.

Solution to the robust least squares problem, which is equivalent to problem (13) when $\theta$ is not constrained to belong to $\mathcal{D}_{\theta}^{\prime}$, is addressed in (El Ghaoui and Lebret, 1997) where the authors show that such a problem is NP-hard in general. Then, they present a semidefinitive relaxation technique to compute a suboptimal solution. Their approach can be extended to deal with the case of $\theta$ in $\mathcal{D}_{\theta}^{\prime}$. Although such a relaxation technique results to be an effective way of dealing with robust least squares problems, it could become rather cumbersome from both the computation time and the memory storage requirements points of view, when the number of measurements $N$ is large and uncertainty affecting the data is highly structured. Thus, in this work the following alternative pointwise estimator is also considered

$$
\hat{\theta}=\arg \min _{\theta \in \mathcal{D}_{\theta}^{\prime}} J(\theta)
$$


which corresponds to the computation of the least squares estimate constrained to the outer-approximation $\mathcal{D}_{\theta}^{\prime}$ of the feasible set $\mathcal{D}_{\theta}$. Since problem (14) derives from problem (13) by neglecting the presence of uncertainty in the functional to be minimized, it is clear that problem (14) can be also interpreted as an alternative way of computing a suboptimal solution of problem (13). Such an estimate can be reliably computed also in the presence of thousands of experimental data. For such a reason estimator (14) should be preferred to estimator (13) when dealing with identification problems with quite large values of $N$

It is worthwhile remarking that the generalization of the presented identification procedure to the case of noisy input signal is straightforward.

\section{Identification of vehicle lateral dynamics from experimental data}

The algorithm presented in Section 3 has been implemented to identify the model of the vehicle lateral dynamics described by (6) and (7), through the experimental data obtained from 13 different manoeuvres performed on dry roads with a passenger car provided by FIAT s.p.a. These manoeuvres include: 4 steering angle steps in the right side with amplitude $30^{\circ}, 50^{\circ}, 70^{\circ}$ and $80^{\circ} ; 4$ steering angle steps in the left side with amplitude $30^{\circ}, 50^{\circ}, 70^{\circ}$ and $80^{\circ} ; 2$ different double lane changes and 3 different steering angle frequency sweeps. The lateral acceleration and the yaw rate are measured by an accelerometer and by a gyroscope respectively, the sideslip angle measurements are collected by a CorrysDatron optical sensor, while the longitudinal velocity is obtained by means of an estimation procedure implemented by FIAT. The given measurement error bounds on $a_{y_{t}}, r_{t}, \beta_{t}$ and $V_{x_{t}}$ are $0.1 \mathrm{~m} / \mathrm{s}^{2}, 0.1^{\circ} / \mathrm{s}, 0.3^{\circ}$ and 2 $\mathrm{km} / \mathrm{h}$ respectively. The steering angle measurements are affected by negligible noises.

\subsection{Identification Set and Validation Set}

The experimental data are split into two different sets: the identification set and the validation set. The parameters of the LPV models of the lateral dynamics are estimated by using only the data in the identification set, while the accuracy of the obtained model is evaluated on the data of the validation set, which are not used during the identification. The identification set includes the following manoeuvres: 2 steering angle steps in the right side of amplitude $50^{\circ}$ and $80^{\circ} ; 2$ steering angle steps in the left side of amplitude $30^{\circ}$ and $70^{\circ} ; 1$ double lane change; 2 frequency sweeps; while the validation set includes: 2 steering angle steps in the right side of amplitude $30^{\circ}$ and $70^{\circ} ; 2$ steering angle steps in the left side of amplitude $50^{\circ}$ and $80^{\circ} ; 1$ double lane change (the one not considered in the identification set); 1 frequency sweep (the one not considered in the identification set).
The number $N_{i d}$ of experimental data in the identification set is 13,280; while the number $N_{v a l}$ of experimental data in the validation set is 8,733 . Note that the two sets are disjoint, that is no manoeuvre is included in both of them. Besides, attention is paid to guarantee that the two data sets include all the different types of the considered manoeuvres. In fact, they contain at least one steering angle step in the left and right side, one double lane change and one frequency sweep. Thus, if a steering angle step in the right (or left) side is included in one set, the steering angle step of the same amplitude is included in the other set, but in the opposite side. Such a split of the data guarantees that, both in the identification stage and in the validation stage, all the considered experimental situations are taken into account.

In order to evaluate the matching between real data $\left(y_{t}\right)$ and estimated data $\left(\bar{y}_{t}\right)$, we consider the mean square errors in the identification data set $\left(M S E_{i d}\right)$ and in the validation data set $\left(M S E_{\text {val }}\right)$, defined as

$$
M S E_{i d}=\sum_{t=1}^{N_{i d}} \frac{\left(y_{t}-\overline{y_{t}}\right)^{2}}{N_{i d}}, M S E_{v a l}=\sum_{t=1}^{N_{v a l}} \frac{\left(y_{t}-\overline{y_{t}}\right)^{2}}{N_{v a l}}
$$

\subsection{Selection of polynomials degrees}

As stated in Section 2.2, coefficients $a_{i}, b_{j}, c_{k}$ and $d_{\nu}$ in (6) and (7) are assumed to be polynomial functions of both the lateral acceleration $a_{y_{t}}$ and the reciprocal of longitudinal velocity $V_{x_{t}}$. Here, the problem of selecting the degree of such polynomial arises. Indeed, as the degree of polynomials $a_{i}, b_{j}, c_{k}$ and $d_{\nu}$ increases, the degrees of freedom of the LPV model increase too, providing a better matching between real data and estimated data in the identification set. However, a model with a large number of degrees of freedom could overfit the data in the identification set, leading to possibly low accuracy of the identified model when tested on the data of the validation set. Besides, as the degree of polynomials $a_{i}$, $b_{j}, c_{k}$ and $d_{\nu}$ increases, the complexity of the identified model increases too. On the basis of such considerations, we have selected the structure of such polynomials by increasing progressively their degree until the identified model provides a satisfactory accuracy level on the validation set. The dynamic model has been identified for the following five different structures of the functions $a_{i}$, $b_{j}, c_{k}$ and $d_{\nu}$ :

S1. linear in both $a_{y_{t}}$ and $V_{x_{t}}^{-1}$;

S2. quadratic in both $a_{y_{t}}$ and $V_{x_{t}}^{-1}$;

S3. cubic in $a_{y_{t}}$ and quadratic in $V_{x_{t}}^{-1}$;

S4. quadratic in $a_{y_{t}}$ and cubic in $V_{x_{t}}^{-1}$;

S5. cubic in both $a_{y_{t}}$ and $V_{x_{t}}^{-1}$.

The mean square error in the validation set $M S E_{v a l}$ for the yaw rate and sideslip angle, identified through the above structures of the functions $a_{i}, b_{j}, c_{k}$ and $d_{\nu}$, 
are reported in Table 1, together with the number of estimated parameters for modeling the vehicle lateral dynamics. By analyzing the results in Table 1, we have chosen the structure $S \mathscr{2}$ for the function $a_{i}, b_{j}, c_{k}$ and $d_{\nu}$, which provides a good tradeoff between accuracy and complexity of the model. Therefore, the assumed structures for $a_{i}, b_{j}, c_{k}$ and $d_{\nu}$ in (6)-(7) are

$$
\begin{aligned}
& a_{i}\left(a_{y_{t}}, V_{x_{t}}^{-1}\right)=a_{i 1}+a_{i 2} a_{y_{t}}+a_{i 3} a_{y_{t}}^{2}+a_{i 4} \frac{a_{y_{t}}}{V_{x_{t}}}+ \\
& +a_{i 5} \frac{a_{y_{t}}^{2}}{V_{x_{t}}}+a_{i 6} \frac{a_{y_{t}}}{V_{x_{t}}^{2}}+a_{i 7} \frac{1}{V_{x_{t}}}+a_{i 8} \frac{1}{V_{x_{t}}^{2}}+a_{i 9} \frac{a_{y_{t}}^{2}}{V_{x_{t}}^{2}} \\
& b_{j}\left(a_{y_{t}}, V_{x_{t}}^{-1}\right)=b_{j 1}+b_{j 2} a_{y_{t}}+b_{j 3} a_{y_{t}}^{2}+b_{j 4} \frac{a_{y_{t}}}{V_{x_{t}}}+ \\
& +b_{j 5} \frac{a_{y_{t}}^{2}}{V_{x_{t}}}+b_{j 6} \frac{a_{y_{t}}}{V_{x_{t}}^{2}}+b_{j 7} \frac{1}{V_{x_{t}}}+b_{j 8} \frac{1}{V_{x_{t}}^{2}}+b_{j 9} \frac{a_{y_{t}}^{2}}{V_{x_{t}}^{2}} \\
& c_{k}\left(a_{y_{t}}, V_{x_{t}}^{-1}\right)=c_{k 1}+c_{k 2} a_{y_{t}}+c_{k 3} a_{y_{t}}^{2}+c_{k 4} \frac{a_{y_{t}}}{V_{x_{t}}}+ \\
& +c_{k 5} \frac{a_{y_{t}}^{2}}{V_{x_{t}}}+c_{k 6} \frac{a_{y_{t}}}{V_{x_{t}}^{2}}+c_{k 7} \frac{1}{V_{x_{t}}}+c_{k 8} \frac{1}{V_{x_{t}}^{2}}+c_{k 9} \frac{a_{y_{t}}^{2}}{V_{x_{t}}^{2}} \\
& d_{\nu}\left(a_{y_{t}}, V_{x_{t}}^{-1}\right)=d_{\nu 1}+d_{\nu 2} a_{y_{t}}+d_{\nu 3} a_{y_{t}}^{2}+d_{\nu 4} \frac{a_{y_{t}}}{V_{x_{t}}}+ \\
& +d_{\nu 5} \frac{a_{y_{t}}^{2}}{V_{x_{t}}}+d_{\nu 6} \frac{a_{y_{t}}}{V_{x_{t}}^{2}}+d_{\nu 7} \frac{1}{V_{x_{t}}}+d_{\nu 8} \frac{1}{V_{x_{t}}^{2}}+d_{\nu 9} \frac{a_{y_{t}}^{2}}{V_{x_{t}}^{2}} .
\end{aligned}
$$

\subsection{Obtained results and discussion}

The comparison between real data and the simulated response provided by the identified LPV model and the single track model described in section 2.1 is reported here. Time-varying velocity is used when simulating the single track model in order to perform a fair comparison with the LPV model. The values of the physical parameters, corresponding to the passenger car used during the experimental test and used in the simulation of the single track model, have been provided by FIAT. Because we are dealing with problem with a large number of experimental data (the length $N_{i d}$ of the identification data set is 13,280 ) the pointwise estimator (14) has been preferred to (13) in the SM-identification of

Table 1

Number of estimated parameters $(p)$ and $M S E_{\text {val }}$ for the yaw rate and sideslip angle description obtained for the five different structures S1-S5 of the functions $a_{i}, b_{j}, c_{k}$ and $d_{\nu}$.

\begin{tabular}{cccc}
\hline $\begin{array}{c}\text { Structure of the } \\
\text { functions } \\
a_{i}, b_{j}, c_{k} \text { and } d_{\nu}\end{array}$ & $\begin{array}{c}M S E_{\text {val }} \\
{\left[\left({ }^{\circ} / \mathrm{s}\right)^{2}\right]}\end{array}$ & $\begin{array}{c}M S E_{\text {val }} \\
{\left[\left(^{\circ}\right)^{2}\right]}\end{array}$ & $p$ \\
\hline$S 1$ & 4.05 & 26 & 20 \\
(yaw rate) & (sideslip angle) & \\
$S 3$ & 0.91 & 0.028 & 45 \\
$S 4$ & 0.87 & 0.023 & 60 \\
$S 5$ & 0.83 & 0.021 & 60 \\
\hline
\end{tabular}

the LPV model for computational-burden reasons. The $M S E_{i d}$ and $M S E_{v a l}$ is computed both for the yaw rate and the sideslip angle and they are reported in Table 2 and Table 3 respectively, which show that the estimated SM-LPV model is significantly more accurate than the single track one in describing the vehicle lateral dynamics. Fig. 1 shows a comparison between the estimated SM-LPV model and the single track one in describing the yaw rate and the sideslip angle for a steering angle step of $70^{\circ}$ in the right side. The same models are compared in Fig. 2 for a double lane change manoeuvre. As expected, the single track model is not able to properly describe the sideslip angle dynamics during manoeuvres such as double lane changes and steering angles steps with amplitude greater than $50^{\circ}$, which highly excite the nonlinearities of the vehicle (see Fig. 1(b) and Fig. 2(b)).

In order to show the effectiveness of the identification procedure presented in this work, performances of the SM-LPV model identified through (14) are also compared with the performances obtained by an LPV model with the same structure, whose parameters $\theta_{L S}$ are obtained without constraining $\theta$ to belong to the feasible set $\mathcal{D}_{\theta}^{\prime}$. This means that parameter $\theta_{L S}$ is computed through the standard LS estimation, i.e.

$$
\theta_{L S}=\arg \min _{\theta}\|\phi \theta-y\|_{2}
$$

The $M S E_{i d}$ and $M S E_{v a l}$ obtained by the LS-LPV model in describing the vehicle lateral dynamics are reported in Table 2 and Table 3. Since in (14) the parameters $\theta$ are constrained to belong to the feasible set $\mathcal{D}_{\theta}^{\prime}$, the mean square error $M S E_{i d}$ provided by the LS-LPV model estimated through (15) is lower than the $M S E_{i d}$ achieved by the SM-LPV model. On the other hand, the $M S E_{\text {val }}$ obtained by the SM-LPV model is lower than the one obtained by the LS-LPV model. These results can be explained by the fact that the LS-estimation (15) does not exploit the information on the noise bounds and tends to overfit the data in the identification set, leading to a less accurate identified model with respect to the SM-LPV model, which, on the contrary, takes into account the prior information on the noise by constraining the LPV parameters $\theta$ to be consistent with given error bounds. Fig. 3 shows the comparison between the SM-LPV model and the LS-LPV model for a steering angle step manoeuvre of $80^{\circ}$ in the left side, which, among the experimented manoeuvres, is the one where the difference between the two models is more evident.

The plot of the two time-varying parameters is shown only for the case of double lane change manoeuvre: the longitudinal velocity $V_{x_{t}}$ is shown in Fig. 4(a) while the lateral acceleration $a_{y_{t}}$ is shown in Fig. 4(b). 


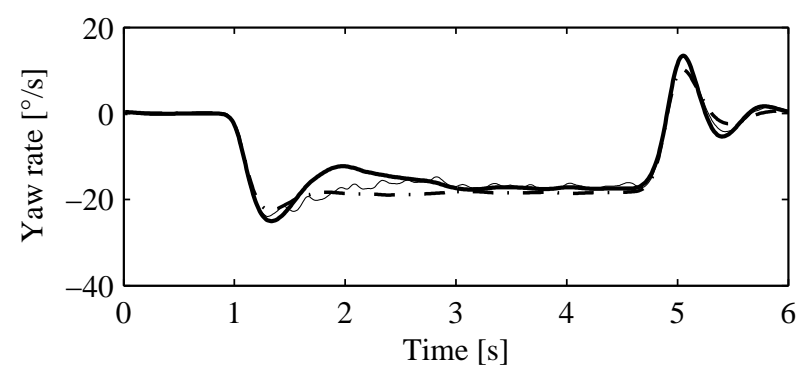

(a)

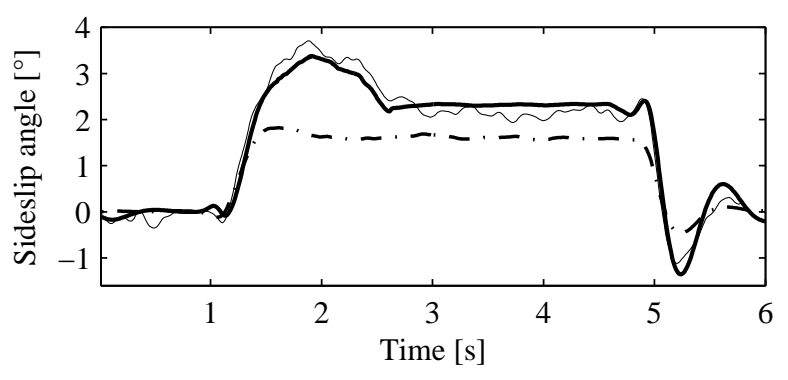

(b)

Fig. 1. Comparison between SM-LPV model and single track model. (a) yaw rate and (b) sideslip angle for steering angle step of $70^{\circ}$ in the right side. Real data (thin), estimated data with SM-LPV model (thick), estimated data with single track model (dashdot).

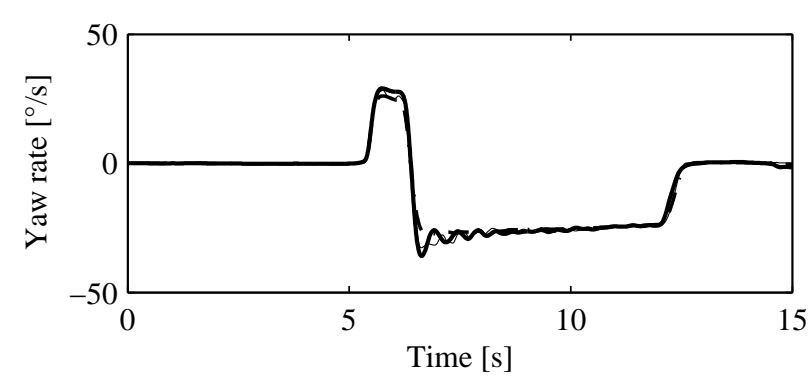

(a)

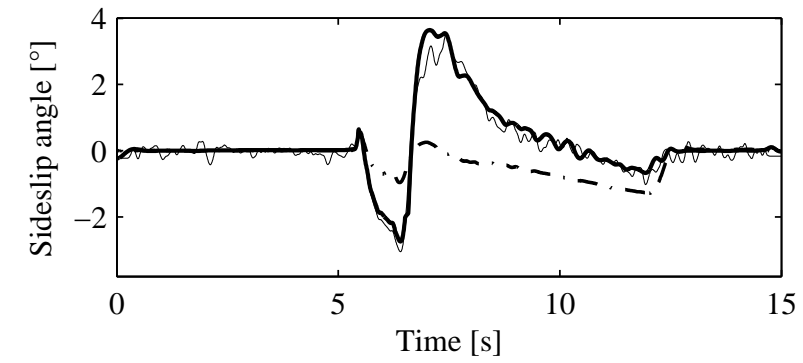

(b)

Fig. 2. Comparison between SM-LPV model and single track model. (a) yaw rate and (b) sideslip angle for double lane change manoeuvre. Real data (thin), estimated data with SM-LPV model (thick), estimated data with single track model (dashdot).

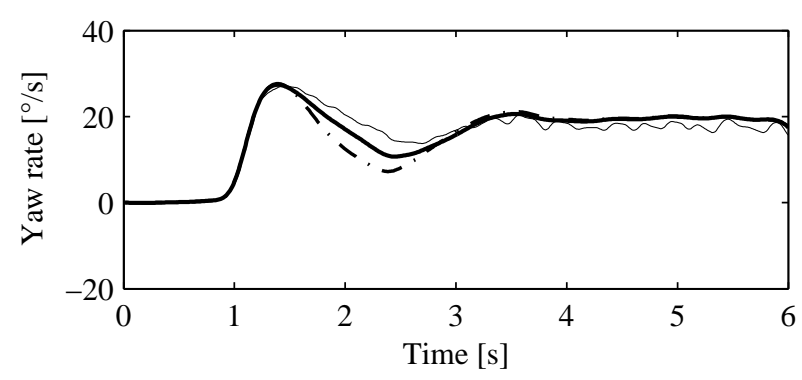

(a)

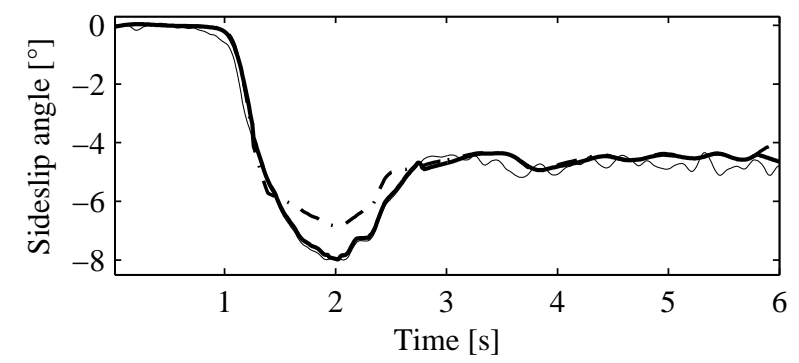

(b)

Fig. 3. Comparison between SM-LPV model and LS-LPV model. (a) yaw rate and (b) sideslip angle for steering angle step of $80^{\circ}$ in the left side. Real data (thin), estimated data with SM-LPV model (thick), estimated data with LS-LPV model (dashdot).

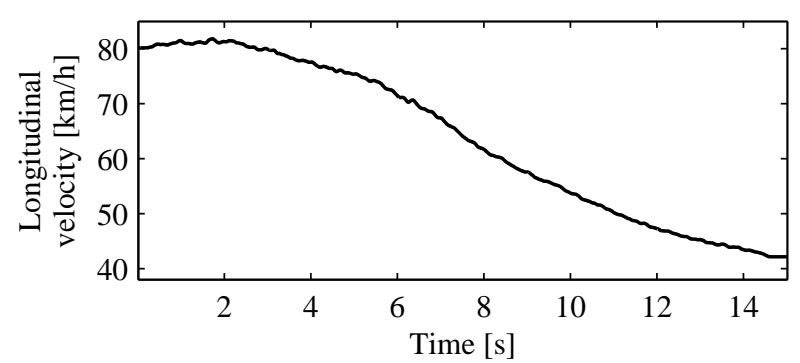

(a)

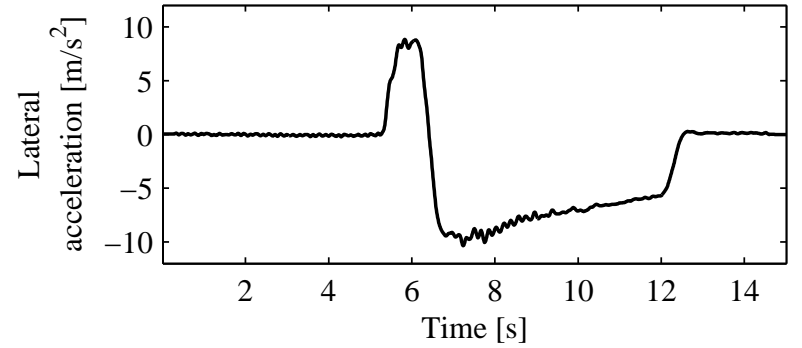

(b)

Fig. 4. Double lane change manoeuvre: (a) longitudinal velocity and (b) lateral acceleration. 
Table 2

MSE in yaw rate description.

\begin{tabular}{ccc}
\hline model & $M S E_{i d}\left[(\% / \mathrm{s})^{2}\right]$ & $M S E_{\text {val }}\left[\left({ }^{\circ} / \mathrm{s}\right)^{2}\right]$ \\
\hline single track & 2.25 & 2.39 \\
\hline SM-LPV (14) & 0.56 & 0.91 \\
\hline LS-LPV (15) & 0.38 & 1.08 \\
\hline
\end{tabular}

Table 3

MSE in sideslip angle description.

\begin{tabular}{ccc}
\hline model & $M S E_{i d}\left[\left(^{\circ}\right)^{2}\right]$ & $M S E_{\text {val }}\left[\left(^{\circ}\right)^{2}\right]$ \\
\hline single track & 0.63 & 1.21 \\
\hline SM-LPV (14) & 0.019 & 0.028 \\
\hline LS-LPV (15) & 0.008 & 0.044 \\
\hline
\end{tabular}

\section{Conclusion}

Set-membership LPV identification of vehicle lateral dynamics is considered in the paper. First, the structure of the model to be identified is properly selected on the basis on physical insights on the vehicle dynamics. Then, a previously published algorithm for the identification of SISO LPV models when both the output and the timevarying measurements are affected by bounded noise is exploited to identify an LPV model of the vehicle lateral dynamics from a large set of experimental data. The obtained results show that the identified LPV model describes the vehicle lateral dynamics more accurately than the single track model and the LPV model identified through the standard LS algorithm.

\section{References}

Belforte, G. and P. Gay (2004). Optimal worst case estimation for lpv-fir models with bounded errors. Systems 86 Control letters 53, 259-268.

Bianchi, F. and R. S. Sanchez-Pena (2010). Robust identification/invalidation in an lpv framework. International Journal of Robust and Nonlinear Control 20(3), 301-312.

Cerone, V. and D. Regruto (2008). Set-membership identification of LPV models with uncertain measurements of the timevarying parameter. In: Proceedings of 47 th Conference on Decision and Control. pp. 4491-4496.

Cerone, V. M. Milanese and D. Regruto (2009a). Combined automatic lane-keeping and driver's steering through a 2dof control strategy. IEEE Transaction on Control Systems Technology 17(1), 135-142.

Cerone, V. M. Milanese and D. Regruto (2009b). Yaw stability control design through a mixed sensitivity approach. IEEE Transaction on Control Systems Technology 17(5), 10961104.

El Ghaoui, L. and H. Lebret (1997). Robust solutions to leastsquares problems with uncertain data. SIAM J. Matrix Appl. 18(4), 1035-1064.

Gaspar, P. Z. Szabo and J. Bokor (2008). A grey-box identification of an lpv vehicle model for observer-based sideslip angel estimation. In: Proceedings of American Control Conference, New York, USA, $200 \%$.
Güvenç, B.A. T. Bünte, D. Odenthal and L. Güvenç (2004). Robust two degree-of-freedom vehicle steering controller design. IEEE Transactions On Control Systems Technology 12(4), 627-636.

Imsland, L. T. A. Johansen, T. I. Fossen, H. F. Grip and J. C. Kalkkuhl (2006). Vehicle velocity estimation using nonlinear observer. Automatica 42, 2091-2103.

Kacewicz, B.Z. M. Milanese, R. Tempo and A. Vicino (1986). Optimality of central and projection algorithms for bounded uncertainty. Systems and Control Letters 8, 161-171.

Milanese, M. Norton, J. Piet-Lahanier, H. and Walter, E. Eds.) (1996). Bounding approaches to system identification. Plenum Press. New York.

Piyabongkarn, D. R. Rajamani, J.A. Grogg and J.Y. Lew (2009). Development and experimental evaluation of a slip angle estimator for vehicle stability control. IEEE Transactions On Control Systems Technology 17(1), 78-88.

Poussot-Vassal, C. O. Sesame, L. Dugard, P. Gáspár and Z. Szabó (2008). A new semi-active suspension control strategy through LPV technique. Control Engineering Practice 16(12), 1519-1534.

Rajamani, R. (2006). Vehicle Dynamics and Control. Springer.

Rajamani, R. and J.K. Hedrick (1994). Performance of active automotive suspensions with hydraulic actuators: theory and experiments. In: Proceedings of the American Control Conference, 1994, Baltimore, Maryland, USA. pp. 12141218.

Rajamani, R. H. S. Tan, B. Law and W. B. Zhang (2000). Demonstration of integrated lateral and longitudinal control for the operation of automated vehicles in platoons. IEEE Transactions On Control Systems Technology 8(4), 695-708.

Rugh, W. J. and J. S. Shamma (2000). Research on gain scheduling. Automatica 36(10), 1401-1425.

Stephant, J. A. Charara and D. Meizel (2004). Virtual sensor: Application to vehicle sideslip angle and transversal forces. IEEE Transactions On Industrial Electronics 51(2), 278-289.

Suryanarayanan, S. M. Tomizuka and T. Suzuki (2004). Design of simultaneously stabilizing controllers and its application to faul-tolreant lane-keeping controller design for automated vehicles. IEEE Transaction on Control Systems Technology 12(3), 329-339.

Sznaier, M. and C. Mazzarro (2003). An LMI approach to control-oriented identification and model (in)validation of LPV systems. IEEE Transaction on Automatic Control 48(9), 1619-1624.

Toth, R. (2010). Modeling and identification of linear parametervarying systems. Springer.

Wang,

$\mathrm{J}$.

Alexander and R. Rajamani (2004). Friction estimation on highway vehicles using longitudinal measurements. ASME J, Dynam. Syst. Meas. Control 126(2), 265-275. 